\title{
Coordinated Control and Estimation of Multiagent Systems with Engineering Applications
}

\author{
Housheng Su, ${ }^{1}$ Michael Z. Q. Chen, ${ }^{2}$ Qing Hui, ${ }^{3}$ Wei Zhang, ${ }^{4}$ and Fanglai Zhu ${ }^{5}$ \\ ${ }^{1}$ School of Automation, Huazhong University of Science and Technology, Wuhan 430074, China \\ ${ }^{2}$ Department of Mechanical Engineering, The University of Hong Kong, Pokfulam Road, Hong Kong \\ ${ }^{3}$ Department of Mechanical Engineering, Texas Tech University, Lubbock, TX, USA \\ ${ }^{4}$ Laboratory of Intelligent Control and Robotics, Shanghai University of Engineering Science, Shanghai 201620, China \\ ${ }^{5}$ College of Electronics and Information Engineering, Tongji University, Shanghai 200092, China
}

Correspondence should be addressed to Housheng Su; houshengsu@qq.com

Received 14 December 2014; Accepted 14 December 2014

Copyright (C) 2015 Housheng Su et al. This is an open access article distributed under the Creative Commons Attribution License, which permits unrestricted use, distribution, and reproduction in any medium, provided the original work is properly cited.

Recently, coordinated control and estimation problems have attracted a great deal of attention in different fields especially in biology, physics, computer science, and control engineering. Coordinated control and estimation problems have prominent characteristics of distributed control, local interaction, and self-organization. Research on multiagent coordinated control and estimation problems not only helps better understand the mechanisms of natural collective phenomena but also benefits the applications of cyberphysical systems.

This special issue focuses on theoretical and technological achievements in cooperative multiagent Systems. It contains twenty-six papers, the contents of which are summarized below.

(1) Coordinated Control of Multiagent Systems and Synchronization of Complex Networks. D. Yang and X. Liu "Distributed Robust Attitude Tracking of Multiple Spacecraft with Disturbances and Unmodelled Dynamics" investigated the distributed robust attitude tracking problem of multiple spacecraft subject to disturbances and unmodelled dynamics using the relative attitudes and relative angular velocities of neighbors. L. Ma et al. "Distributed Multiagent Control Approach for Multitarget Tracking" presented a novel control approach in distributed manner for multitarget tracking by providing a suboptimal multiagent control solution by maximizing the local Rényi divergence. F. Jiang et al. "Extremum Seeking Based Fault-Tolerant Cooperative Control for Multiagent Systems" proposed a novel fault tolerant cooperative control strategy for multiagent systems, and a real-time adaptive extremum seeking algorithm is utilized for adaptive approximation of fault parameter.

H. Zhou et al. "Flocking Control of Multiple Mobile Agents with the Rules of Avoiding Collision" investigated the flocking and the coordinative control problems of multiple mobile agents with the rules of avoiding collision. L. Wang et al. "Adaptive Synchronization via State Predictor on General Complex Dynamic Networks" discussed the adaptive synchronization of general complex dynamic networks via state predictor based on the fixed topology for nonlinear dynamical systems. W. Hu et al. "Impulsive Containment Control in Nonlinear Multiagent systems with Time-Delay" studied the containment control problems of nonlinear multiagent systems with time-delay via impulsive algorithms under both fixed and switching topologies. J. Ma et al. "Distributed Event-Triggered Control of Multiagent Systems with TimeVarying Topology" investigated the consensus of first-order discrete-time multiagent systems with time-varying interaction topology. C. Xu et al. "Pinning-Like Adaptive Consensus for Networked Mobile Agents with Heterogeneous Nonlinear Dynamics" studied the adaptive consensus for networked mobile agents with heterogeneous nonlinear dynamics. B. Liu et al. "Consensus of the Multiagent System with a Dynamic Leader Based on Directed Topology Using Laplace Transform" investigated the consensus of the multiagent system with directed topology and a dynamic leader. B. Liu et al. "Consensus of Multiagent Systems with Directed Topology and Communication Time Delay Bases on the 
Laplace Transform" investigated the consensus problem of multiagent systems with directed topologies by proposing a new method, the Laplace transform, to study the consensus of multiagent systems. Y.-J. Sun et al. "Consensus Analysis for a Class of Heterogeneous Multiagent Systems with Time Delay Based on Frequency Domain Method" investigated the consensus problem of heterogeneous multiagent systems composed of first-order and second-order agent based on frequency domain method. The sufficient consensus conditions were obtained. M. Yu et al. "Average Consensus in Multiagent Systems with the Problem of Packet Losses When Using the Second-Order Neighbors' Information" investigated the average consensus of multiagent systems with the problem of packet losses when both the first-order and the second-order neighbors' information are used.

(2) Distributed Estimation and Control for Mobile Sensor Networks. Y. Bo et al. "Improved Different Dimensional Sensors Combined Space Registration Algorithm" studied a method based on the state value and space deviation of federated filtering of unscented Kalman filter and standard Kalman filter, which conduces to real time registering of system deviation of radar and IF sensors. B. Yan et al. "Robust Fault Detection for a Class of Uncertain Nonlinear Systems Based on MultiObjective Optimization" presented a robust fault detection scheme for a class of nonlinear systems with uncertainty. The proposed approach utilizes robust control theory and parameter optimization algorithm to design the gain matrix of fault tracking approximator (FTA) for fault detection. The design of the gain matrix of FTA takes into account the robustness of residual signals to system uncertainty and sensitivity of residual signals to system faults simultaneously, which leads to a multiobjective optimization problem. Then, the detectability of system faults is analyzed by investigating the threshold of residual signals. W. Liu et al. "Energy-Efficient Node Scheduling Method for Cooperative Target Tracking in Wireless Sensor Networks" proposed an energy-efficient node scheduling method is proposed to minimize energy consumption while ensuring the tracking accuracy. The proposed scheduling method can keep the tracking accuracy while minimizing energy consumption and the NP-complete nature is avoided. B. Yan et al. "Distributed Fault Detection for a Class of Nonlinear Stochastic Systems" presented a novel distributed fault detection strategy for a class of nonlinear stochastic systems, in which a nonlinear fault detection filter was constructed to provide estimation of unmeasurable system states and residual signals using outputs of the consensus filter.

(3) Applications of Coordinated Control of Complex Networked Systems. L. Qin et al. "Distributed Multiagent for NAO Robot Joint Position Control Based on Echo State Network" studied the joints position control of NAO robot. X. Dongdong et al. "The Study on Detection Method of Water Vapor on Boundary Layer Based on Multiagent System" proposed a method of detecting water vapor on boundary layer based on multiagent system. G. Chen et al. "A Core Model for Parts Suppliers Selecting Method in Manufacturing Supply Chain" proposed a core model for parts suppliers selecting method in manufacturing supply chain. H. Zhang et al. "Data Fusion Based Thermal Sensors for Mass Flow Measurement in Pneumatic Conveying" investigated the mass flow measurement of the gas-solid two-phase flow in pneumatic conveyor and proposed a new data fusion method based on the thermal sensors. D. Wang and S. Wu "Design of the Congestion Control for TCP/AQM Network with Time-Delay” designed congestion controller for TCP/AQM (transmission control protocol/Active queue management) networks using model following control, the equilibrium of a class of TCP/AQM network with time-delay was investigated, and the effect of communication time-delay on the stability was addressed. C. Zhang et al. "Hybrid Structure Based Tracking and Consensus for Multiple Motors" investigated a hybrid structure based synchronous control strategy for multimotor system of shaftless-driven printing press. S. Deng et al. "Solving the Fuzzy Bilevel Linear Programming with Multiple Followers through Structured Element Method" showed that the fuzzy bilevel linear programming with multiple followers (MFFBLP) model optimal solution was equivalent to the optimal solution of the bilevel linear programming with multiple followers by using fuzzy structured element theory. W. Zeng et al. "Risk-Sensitive Multiagent DecisionTheoretic Planning Based on MDP and One-Switch Utility Functions" studied multiagent decision-theoretic planning under Markov decision processes (MDPs) framework with considering the change of agent's risk attitude as his wealth level varies. C. Xie and J. Ren "A Dynamical Reliability Prediction Algorithm for Composite Service" proposed a new reliability predicting algorithm for composite services. Comparing with the traditional reliability model, the new dynamic reliability approach is more flexible, which does not recompute reliability for all composite units and only computes the reliability of the effected composite units. D. Sun and X. Kou "Punishment Effect of Prisoner Dilemma Game Based on a New Evolution Strategy Rule" discussed the effect of the punishment in the Prisoner's Dilemma Game and proposed a new evolution strategy rule which can reflect the external factor for both players in the evolution game.

Note that the selected topics and papers are not a comprehensive representation of the area covered by the special issue. Note, however, that the published papers in this special issue do provide some recent advances in the field of multiagent systems, which could benefit the current research in some way.

\section{Acknowledgments}

We would like to thank all the authors for their contributions and acknowledge all the reviewers for their time and effort in assessing the manuscripts.

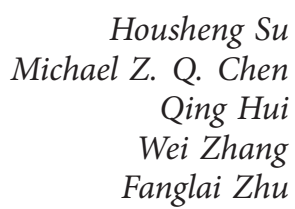




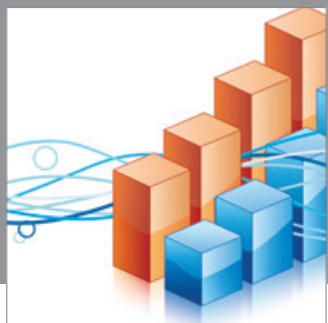

Advances in

Operations Research

mansans

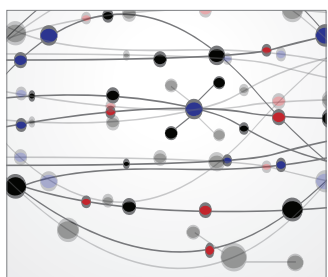

The Scientific World Journal
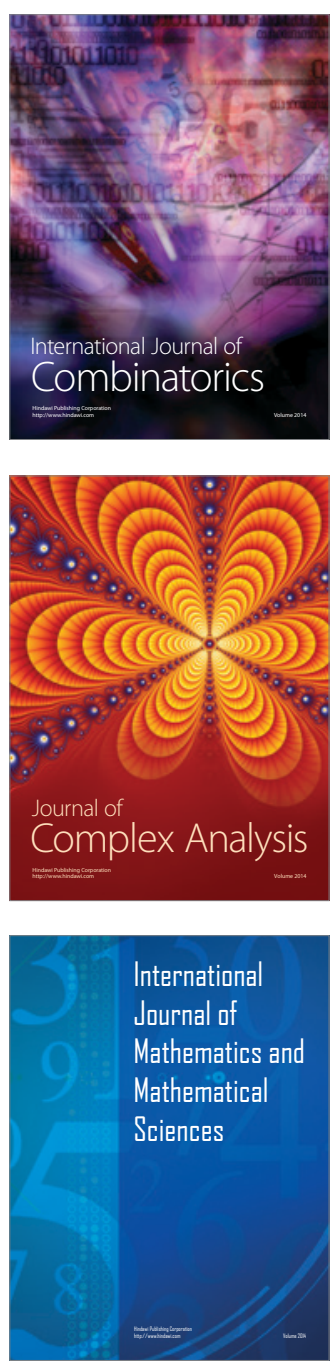
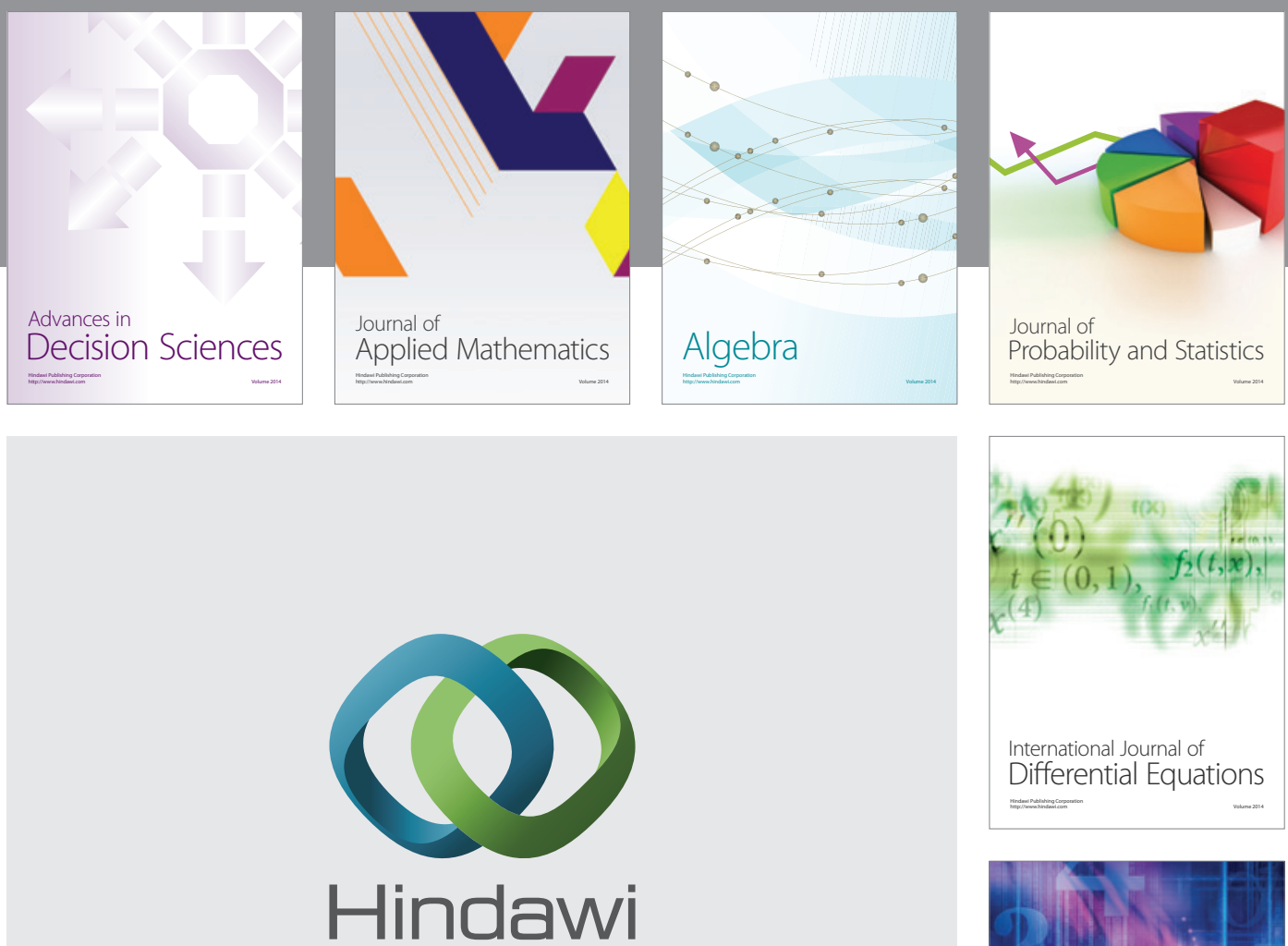

Submit your manuscripts at http://www.hindawi.com
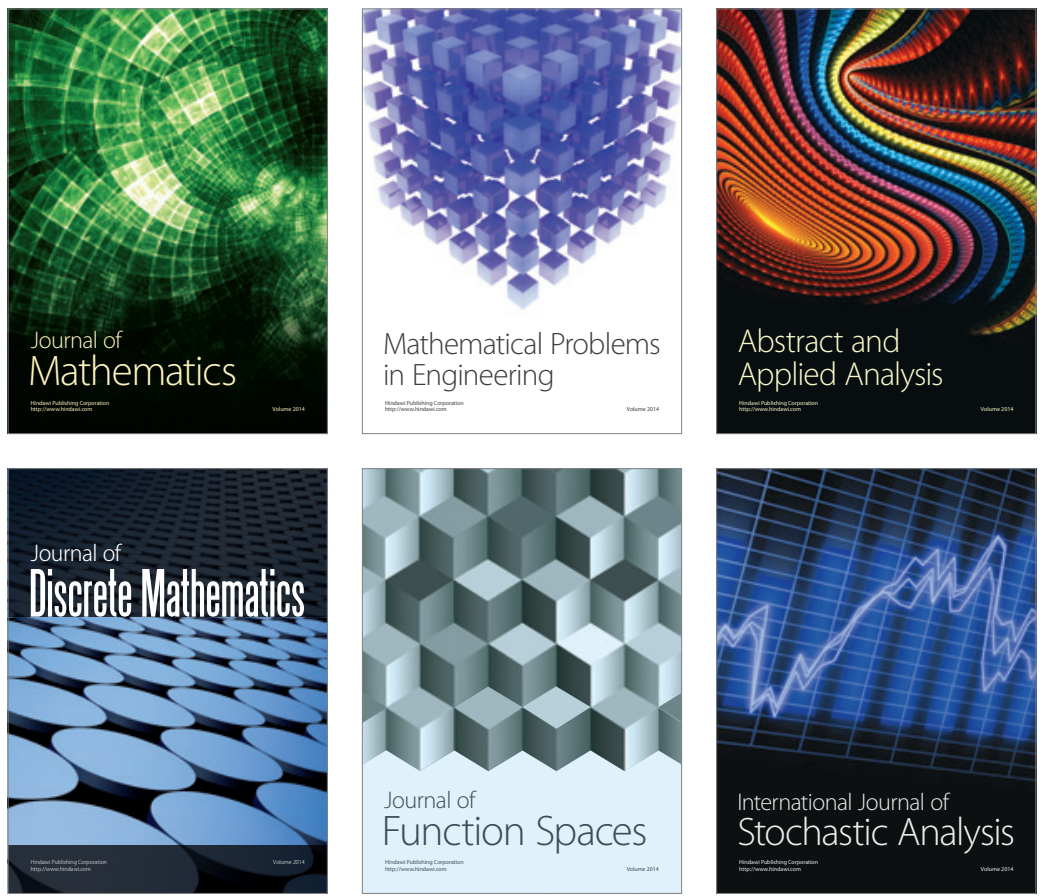

Journal of

Function Spaces

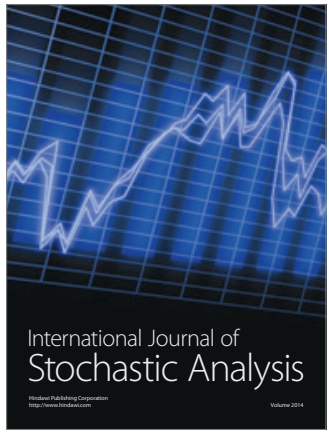

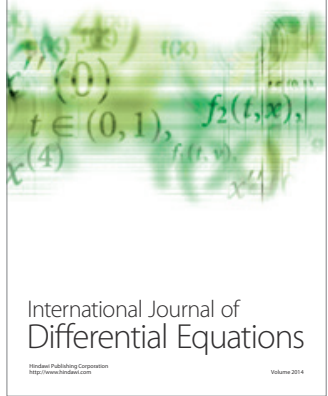
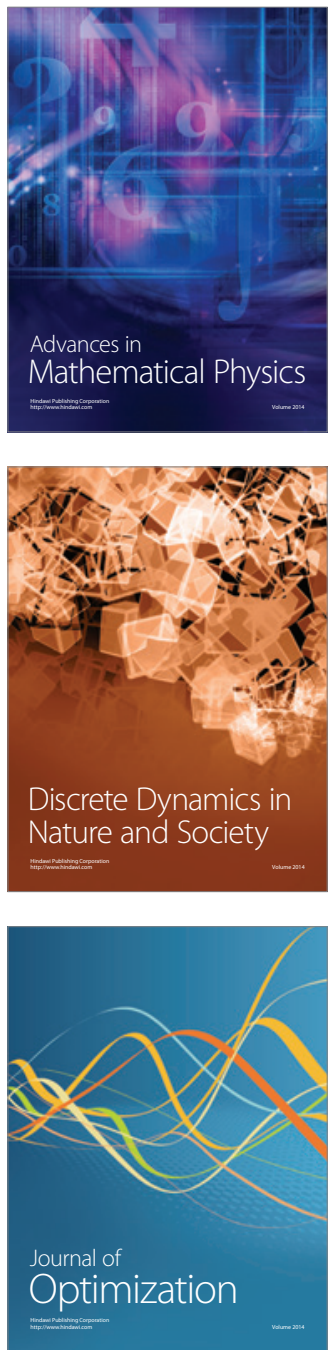